16. Tochel C, Hosey M T, Macpherson L, Pine C. Assessment of children before dental extractions under general anaesthesia in Scotland. Br Dent J 2004; 196: 629-633.

17. Macpherson L M, Pine C M, Tochel C, Burnside G, Hosey $M$ T, Adair P. Factors influencing referral of children for dental extractions under general and local anaesthesia. Community Dent Health 2005; 22: 282-288.

18. Tomlinson D, von Baeyer C L, Stinson J N, Sung L. A systematic review of faces scales for the self-report of pain intensity in children. Paediatrics 2010; 126: E1168E1198.

19. Vernon D T, Schulman J L, Foley J M. Changes in children's behaviour after hospitalization: some dimensions of response and their correlates. Am J Dis Child 1966; 111: 581-593.

20. Newton J T, Sturmey P. Development of a short form of the Treatment Evaluation Inventory for the acceptability of psychological interventions. Psychol Rep 2004; 94 : 475-481.
21. NICE British National Formulary. Fentanyl. Available online at https://bnf.nice.org.uk/drug/fentanyl.html accessed 16 September 2019).

22. Acs G, Pretzer S, Foley M, Ng M W. Perceived outcomes and parental satisfaction following dental rehabilitation under general anaesthesia. Paediatr Dent 2001; 23: 419-423.

23. Fung D E, Cooper D J, Barnard K M, Smith P B. Pain reported by children after dental extractions under general anaesthesia: a pilot study. Int J Paediatr Dent 1993; 3: 23-28.

24. Gazal G, Mackie I C. Distress related to dental extraction for children under general anaesthesia and their parents. Eur J Paediatr Dent 2007: 8: 7-12.

25. Atan S, Ashley P, Gilthorpe M S, Scheer B, Mason C, Roberts $\mathrm{G}$. Morbidity following dental treatment of children under intubation general anaesthesia in a day-stay unit. Int J Paediatr Dent 2004; 14: 9-16.
26. Rodd H, Hall M, Deery C, Gibson B J, Marshman Z. "I felt weird and wobbly." Child-reported impacts associated with a dental general anaesthetic. Br Dent 2014; 216: 1-5.

27. Anderson H K, Drummond B K, Thomson W M. Changes in aspects of children's oral-health-related quality of life following dental treatment under general anaesthesia. Int J Paediatr Dent 2004; 14: 317-325.

28. Jankauskiene B, Narbutaite J. Changes in oral healthrelated quality of life among children following dental treatment under general anaesthesia. A systematic review. Stomatologija 2010; 12: 60-64.

29. Huntington C, Newton J T, Donaldson $\mathrm{N}$ et al. Lessons learned on recruitment and retention in hardtoreach families in a phase III randomised controlled trial of preparatory information for children undergoing general anaesthesia. BMC Oral Health 2017; 17: 122.

\title{
Correction to: Interceptive extractions for first permanent molars: a clinical protocol
}

The original article can be found online at https://doi.org/10.1038/s41415-019-0561-7.

Author's correction note:

Clinical article Br Dent J 2019; 227: 192-195.

When this article was initially published, one of the boxes in Figure 2 had been duplicated. The correct figure is shown below:

\section{Fig 2 Flowchart of first permanent molar management}

This is intended as guidance only and should not be viewed as prescriptive for treatment.

Patient presents with one or more compromised FPMs that do not require immediate extraction and is prepared to consider interceptive extraction of the FPM. The SPM is unerupted.

- Consider the most seriously affected tooth first

- Consider each side separately as FPM interceptive extractions are not balanced.

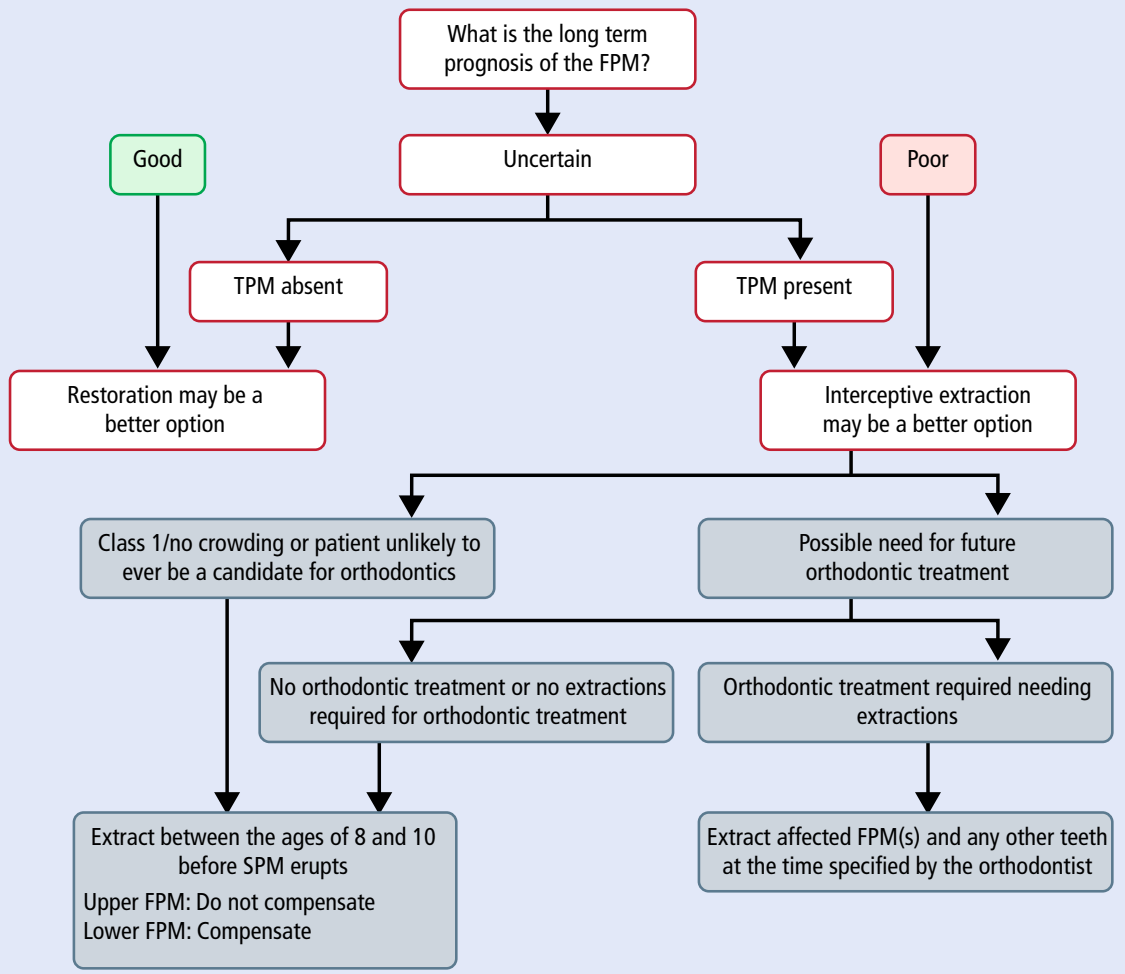

The journal apologises for any confusion caused by this error. 